\title{
Mechanical vibrations of magnetically levitated viscoelastic droplets
}

\author{
Robert H. Temperton, Richard J.A. Hill, and James S. Sharp* \\ Received Xth $X X X X X X X X X X 20 X X$, Accepted Xth $X X X X X X X X X 20 X X$ \\ First published on the web Xth $X X X X X X X X X X 200 X$ \\ DOI: 10.1039/b000000x
}

The mechanical vibrations of magnetically levitated droplets were investigated using a simple optical deflection technique. Droplets of water and a water-based solution of poly(acrylamide-co-acrylic acid ) were levitated in the bore of a superconducting magnet and perturbed with a short puff of air. Centre of mass and surface vibrations were monitored using laser light refracted through the droplet, focussed on to the end of an optical fiber and detected using a photodiode. Time dependent variations in the voltage generated by the photodiode were Fourier transformed to obtain the frequency and spectral width of the drops' mechanical resonances. A simple theory of drop vibration was developed to extract the rheological properties of the droplets from these quantities. The resulting values of $G$ ' and $G$ " that were extracted were found to be in good agreement with values obtained using conventional rheology techniques.

\section{Introduction}

The frequencies and spectral widths of the normal modes of a Newtonian liquid droplet are well known ${ }^{1,2}$. However, there is little experimental data and/or theory describing the oscillations of an isolated viscoelastic droplet. Additives such as polymers and small particles can have a dramatic effect on the surface and rheological properties of small liquid droplets. These can, in turn, influence properties such as the wetting, spreading and dewetting behaviour of the fluids ${ }^{3-6}$. They also have a significant influence on their performance in applications such as droplet atomisation, ink jet printing, fuel injection $^{7}$, microscale mixing/demixing ${ }^{8,9}$, and during drop impact and rebound phenomena ${ }^{6}$. Viscoelastic vibrational modes may also influence the dynamical properties of atomic nuclei ${ }^{10}$, crustal deformation of planets ${ }^{11,12}$ and energy relaxation mechanisms in neutron stars ${ }^{13}$.

Vibration of small liquid and viscoelastic droplets provides a potentially attractive route for determining parameters such as the surface tension and mechanical properties of materials where sample volumes are limited. Previous studies have successfully used isolated drops to extract values for the surface tension and viscosity of liquid drops. However, little theoretical or experimental work has been performed on viscoelastic drops $^{14-19}$.

In this article we demonstrate that the vibrational frequencies and spectral widths of the mechanical oscillations of viscoelastic droplets of polymer solutions can be used to extract values of the frequency-dependent storage and loss moduli $\left(G^{\prime}\right.$ and $G^{\prime \prime}$ respectively). We also derive a heuristic model

School of Physics and Astronomy and Nottingham Nanoscience Centre, University of Nottingham, Nottingham, UK. Fax: +44 (0)115 9515180; Tel: +44 (0)115 9515142; E-mail: james.sharp@nottingham.ac.uk to describe the vibration of free/levitated viscoelastic droplets that can be used to extract these quantities. To the best of our knowledge this study represents the first combined experimental and theoretical study of the vibration of isolated viscoelastic droplets. As such it represents a significant step forward in our understanding of the drop vibration phenomenon and our ability to measure the rheological properties of polymer solutions where the sample volumes are small $(\sim \mu l$ to $m l)$.

\section{Experimental}

Solutions of poly(acrylamide-co-acrylic acid) (PAA, $\mathrm{M}_{w}=15$ MDa, Sigma, UK) were prepared in deionised water (produced by an ELGA purifier) and made up to concentrations of $0,0.5$ and $1 \mathrm{wt} \%$ respectively. The overlap concentration for this polymer ${ }^{20}$ is $\sim 0.1 w t \%$ and as such both of the polymer solutions studied are likely to contain chains that are entangled. Droplets of these solutions were suspended individually in the bore of a $16 \mathrm{~T}$ superconducting solenoid magnet (Oxford Instruments) using diamagnetic levitation. In this technique, a spatially-varying magnetic field exerts an approximately uniform upward body force on a diamagnetic liquid, strong enough to balance its weight while retaining a spherical drop shape. The vertical component of the magnetic force per unit volume is given by $F=\chi \frac{B}{\mu_{o}} \frac{\partial B}{\partial z}$, where $B$ is the magnitude of the magnetic field, $\chi$ is the magnetic volume susceptibility (which is approximately that of water in our experiments, $\left.-9.0 \times 10^{-6}\right), \mu_{o}$ is the permeability of free space and $z$ is the vertical displacement in the magnet bore. The droplets of polymer solution levitated at a critical value of $B \frac{\partial B}{\partial z} \approx 1370 T^{2} m^{-1}$. Further details of the technique, including the mechanical stability of the levitation, are described in detail elsewhere ${ }^{21-25}$. 
Vibrations were excited in the droplets by applying an impulse in the form of a short puff of air which had a duration of $\sim 1 s$ and a pressure of $\sim 1-10 P a$. This was delivered via a $4 \mathrm{~mm}$ diameter nozzle inside the magnet bore placed within $\sim 5 \mathrm{~cm}$ of the droplet. The resulting centre of mass and surface vibrations caused by the application of the impulse were detected optically: a laser beam was passed through the droplets and time dependent variations in the scattered laser intensity were measured using a photodiode connected to a PC via a National Insturments data acquisition card(see figure 1). The incident and scattered light were delivered to and collected from the droplet using optical fibers.

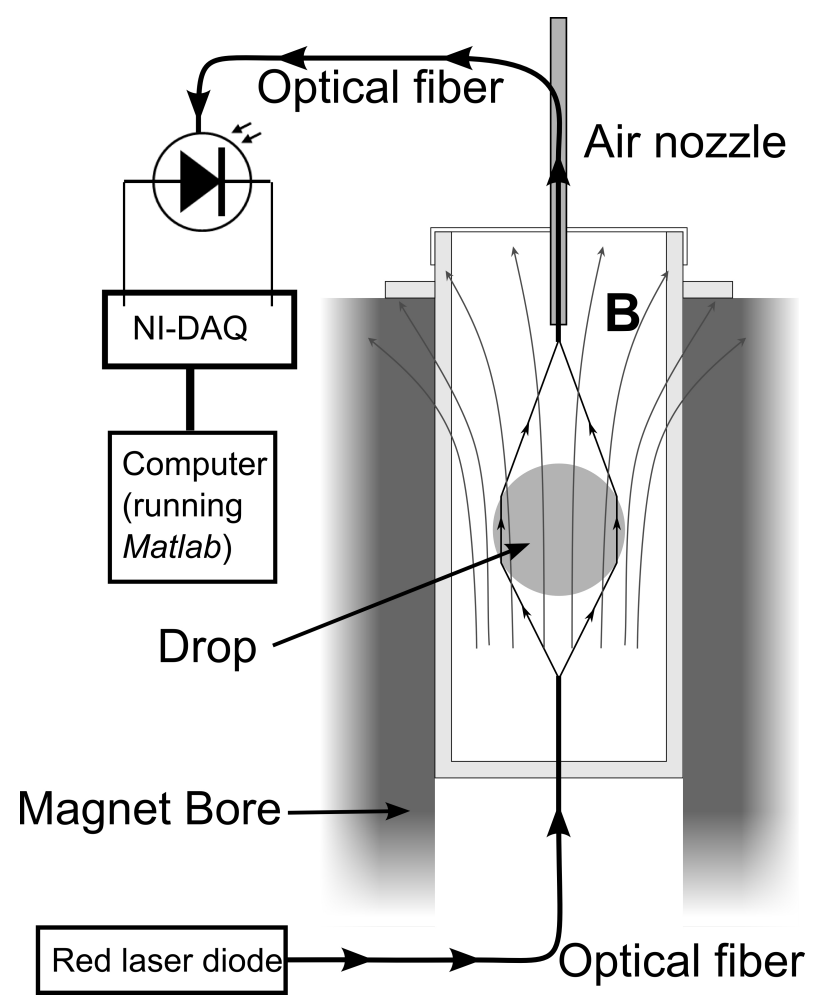

Fig. 1 Diagram of the experimental setup. PAA droplets were levitated in the bore of a super conducting magnet. A puff of air was used to vibrate the droplets and vibrations were detected by measuring temporal changes in the intensity of scattered laser light.

Figure 2 shows an example of a set of results obtained from a $0.98 \mathrm{~g}$ droplet of $0.5 \mathrm{wt} \%$ PAA in water. The intensity variations in the top panel show that the droplets experience low frequency vibrations which correspond to centre of mass oscillations and higher frequency vibrations that are associated with shape changes (surface vibrational modes) in the drops. The acquisition time used to collect the data was fixed at 5 seconds for all the droplets studied. For longer times, viscous damping effects reduced the amplitudes of the surface oscillations below the level that can be distinguished from the noise.
However, it was often necessary to wait for large amplitude centre of mass oscillations (caused by placing the droplets in the bore) to die down before the drops were excited with the impulse and surface vibrations could be detected. As a result of this, droplets were held in the bore of the magnet for 1015 minutes before each experiment. Evaporation losses were minimised by sealing the ends of the magnet bore during this period. Estimates of the mass loss during this time were obtained from measurements of the mass of the pipette before and after placing the drop in the bore and also from the final mass of the drop (which was obtained by soaking the drops on to a small piece of paper towel). These measurements indicate that the mass loss due to evaporation was in the range $2-6 \%$ for the most volatile drops i.e. water.
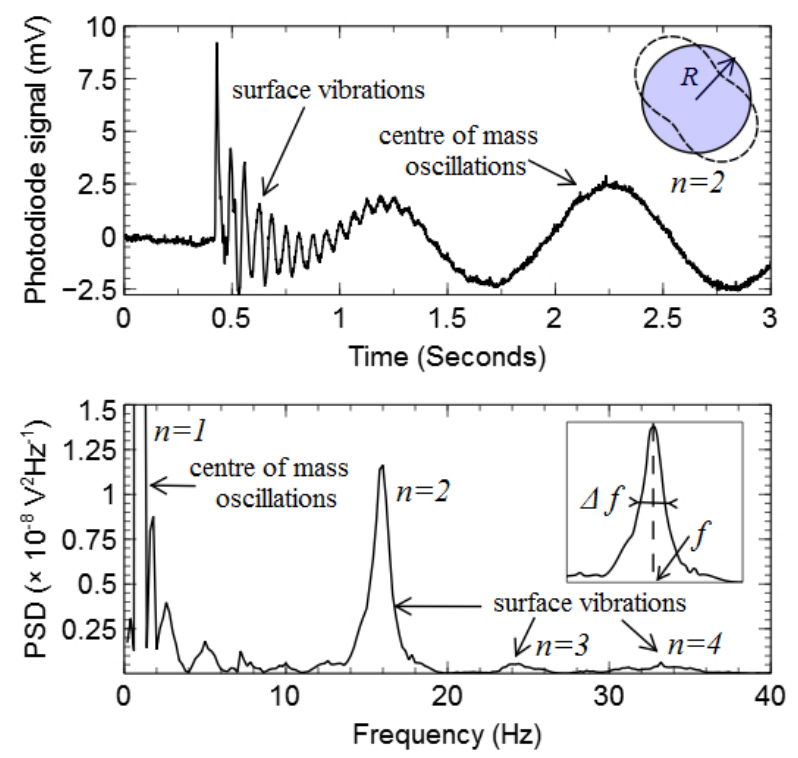

Fig. 2 Vibrational response of PAA loaded droplets. The top panel shows a plot of the temporal response of intensity variations measured using the photodiode for a $0.98 \mathrm{~g}$ droplet of $0.5 \mathrm{wt} \%$ PAA. The bottom panel shows the vibrational spectrum of the droplet obtained by Fourier transformation of the data shown in the top panel. In both panels, features due to centre of mass oscillations and surface vibrations of the droplets are highlighted. The top panel inset shows the expected shape of the lowest $(n=2)$ surface vibrational mode. The bottom panel inset shows how the frequency, $f$, and width, $\Delta f$, of the mechanical resonances are defined.

Fourier transformation of the photodiode signal shown in the top panel of figure 2 gives the mechanical vibrational spectrum of the droplet (bottom panel). The frequency and spectral width (hereafter referred to as the width), which we quantify as the full width at half maximum, were then extracted (see inset in figure 2 for the definition of these quantities). This process was repeated for droplets of different size and composition. Measurements of the density of the PAA solutions 
were obtained by weighing known volumes of the solutions. The radius of each droplet was then determined from its mass and density. Photographs of pendant droplets of the solutions were used to extract the surface tension of the PAA solutions using the well known pendant drop technique ${ }^{26}$ (see inset in figure 3 ).

\section{Results and Discussion}

Figure 3 shows how the frequency and width of the lowest vibrational peak $(n=2)$ vary with the droplet radius for water and a $1 \mathrm{wt} \%$ solution of PAA. As anticipated, the frequency of vibration and width of the vibrational peaks decreases with increasing drop size. The solid line in the top panel of this figure shows the predictions of the vibrational frequencies of the $n=2$ mode for liquid water drops. This was obtained using the equation ${ }^{1}$

$$
f=\sqrt{\frac{n(n-1)(n+2) \gamma}{4 \pi^{2} \rho R^{3}}}
$$

where $\rho$ and $\gamma$ are the density and surface tension of the liquid droplet respectively. The data shown in figure 3 is in excellent agreement with this equation assuming values of $\rho=1000 \mathrm{kgm}^{-3}$ and $\gamma=72 \mathrm{mJm}^{-2}$ for water.

Our analysis of the polymer solution droplets is limited to the study of the two lowest vibrational modes ( $n=2$ and 3$)$ because larger viscous damping effects in the droplets for higher order vibrational modes made it difficult to extract $f$ and $\Delta f$ values for these peaks.

In the absence of a full theory for the normal mode frequencies of a viscoelastic droplet we adopt a heuristic approach in relating the rheological properties of the liquid to the measured vibrational frequencies and widths of the droplet: we consider the dispersion of capillary waves on the surface of a semi-infinite viscoelastic medium with surface tension, as described by Pleiner et. $a l^{27}$,

$$
4 \pi^{2} \rho(f+i \Delta f)^{2}=\frac{4 i(f+i \Delta f)\left(G^{\prime \prime}(f)-i G^{\prime}(f)\right) k^{2}}{f}+\gamma k^{3}
$$

where $\rho$ and $\gamma$ are again the density and surface tension of the viscoelastic medium, $G^{\prime}$ and $G$ ' are the frequency dependent shear storage and loss moduli respectively, $f$ and $\Delta f$ are the frequency and spectral width of the vibrations and $k$ is the magnitude of the surface wavevector. This last quantity is related to the wavelength of the surface vibrations, $\lambda$, by $k=\frac{2 \pi}{\lambda}$.

The semi-infinite medium assumption is justified here because the radius of the droplets is typically an order of magnitude larger than the observed amplitudes of vibration. For the range of droplet radii studied (3-9 $\mathrm{mm}$ ), the amplitudes were typically observed to be less than $0.3-0.9 \mathrm{~mm}$ respectively.

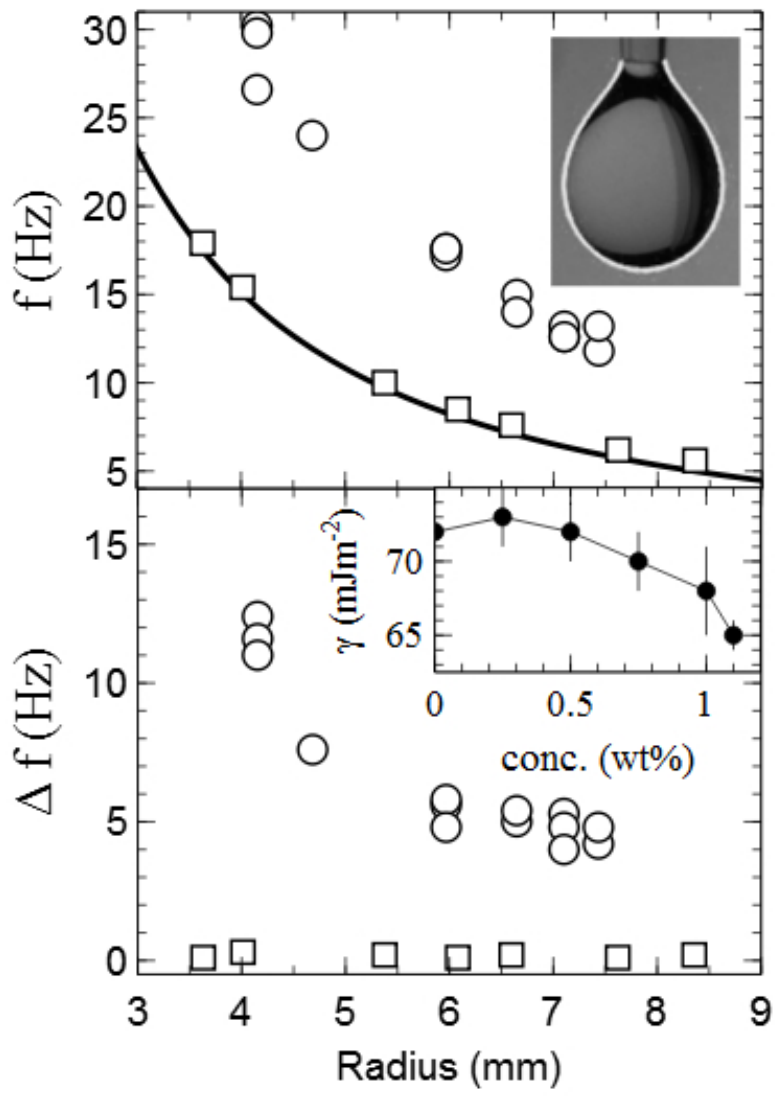

Fig. 3 Radius dependence of droplet vibrational properties. The top panel shows measured variations in the vibrational frequencies of droplets extracted for the $n=2$ vibrational mode. The bottom panel shows the corresponding variation in the width of the mechanical resonances. Data are shown for water $(\square)$ and a $1 \mathrm{wt} \%$ PAA solution $(\bigcirc)$ respectively. The solid line in the top panel shows the predictions of equation 1 for the $n=2$ mode of a water droplet ${ }^{1}$. The top panel inset shows a typical photograph of a pendant drop of a $1 \mathrm{wt} \%$ PAA solution used to extract the surface tension. The diameter of the end of the pipette shown in this image is $0.7 \mathrm{~mm}$ and the white line shows the fit to the drop profile shape obtained using the method outlined in ${ }^{26}$. The bottom panel inset shows the concentration dependence of the surface tension of PAA solutions obtained from similar images. 
The magnetogravitational trap in which the droplet levitates, exerts an effective gravitational force on the drop which acts normal to its surface, giving rise to an effective gravitational acceleration of magnitude $g_{\text {eff }} \sim 0.01 g$. Under these conditions, the capillary length $l_{\text {cap }}=\sqrt{\frac{\gamma}{\rho g_{\text {eff }}}} \approx 2 \mathrm{~cm}$, which is $\sim 2-10 \times$ larger than the radius of the droplets in our experiments. The reduced gravitational contribution to the forces exerted on the droplets results in a slight increase in the frequencies of the normal modes of vibration ${ }^{24}$. However, for the viscoelastic droplets studied here, this correction is small compared to the experimental uncertainty in the measurement of the vibration frequency.

The allowed values of the wavevector, $k$, of surface vibrations at resonance are determined by the requirement that an integer number of vibrational wavelengths, $\lambda$, fit around the periphery of the droplet. This gives the result that $n \lambda=2 \pi R$, where $n$ is a mode number and $R$ is the radius of the drop. We therefore obtain values of $k=\frac{2 \pi}{\lambda}=\frac{n}{R}$ for the allowed surface wavevector magnitudes. Inserting this result into equation 2 and equating real and imaginary parts gives the following expressions for the storage and loss moduli

$$
\begin{aligned}
G^{\prime} & =\frac{\pi^{2} \rho f^{2} R^{2}}{n^{2}}\left(1-\frac{\gamma n^{3}}{4 \pi^{2} \rho R^{3}\left(\Delta f^{2}+f^{2}\right)}\right) \\
G^{\prime \prime} & =\frac{\pi^{2} \rho f \Delta f R^{2}}{n^{2}}\left(1+\frac{\gamma n^{3}}{4 \pi^{2} \rho R^{3}\left(\Delta f^{2}+f^{2}\right)}\right)
\end{aligned}
$$

in the limit when $a=\left|\frac{\rho(f+i \Delta f) f R^{2}}{4 \pi^{2} n^{2}\left(G^{\prime}-i G^{\prime}\right)}\right|<<1$. Inserting typical values of $G^{\prime} \sim G^{\prime \prime} \sim 10 P a, f=30 H z, \Delta f=15 \mathrm{~Hz}, n=2$, $R=3 \mathrm{~mm}, \rho=1000 \mathrm{kgm}^{-3}$ and $\gamma=72 \mathrm{mJm}^{-2}$ we obtain a value of $a=0.004$ for the droplets studied here.

In the case of a Newtonian liquid droplet, where $G^{\prime}=0$, combining equations 3 and 4 gives an expression for the width of the resonance, $\Delta f=\frac{\eta}{\pi \rho R^{2}} n^{2}$, where the viscosity $\eta=\frac{G}{2 \pi f}$. This compares favourably with the exact solution for a viscous sphere, $\Delta f=\frac{\eta}{2 \pi \rho R^{2}}(n-1)(2 n+1)$, derived by Chandrasekhar ${ }^{2}$. The only discrepancy lies in the dependence of the width on the mode number $n$. In the case of the heuristic model presented here, the width scales as $\frac{\eta}{\rho R^{2}}$ multiplied by factors of 4 and 9 for the lowest modes $(n=2$ and $n=3)$. However, the full solution predicts multiplicative factors of 2.5 and 7 for these two modes. For higher modes $(n>3)$, the multiplicative factors predicted by the two theories start to converge as $(n-1)(2 n+1) / 2 \rightarrow n^{2}$ in the limit of large $n$.

Reinserting the result for the width into equation 3 gives an expression for the frequency of vibration of the drops of the form

$$
f=\sqrt{\frac{\gamma n^{3}}{4 \pi^{2} \rho R^{3}}-\frac{\eta n^{2}}{\pi \rho R^{2}}}
$$

which has a similar form that predicted for an inviscid liquid drop (equation 1) with a correction for viscous damping. If we use a typical value for the viscosity of water $\eta=1 \mathrm{mPas}$ this function predicts values for the frequency of vibration that are within $0.04 \%$ of those predicted by equation 1 . As such, a plot of this function would be indistinguishable from the plot of equation 1 shown in figure 3 .

For the case of a perfectly elastic sphere with no dissipation, we would expect surface tension effects to be negligible and the loss modulus to be zero , i.e. $\gamma=0$ and $G "=0$. This predicts that the width of the resonance, $\Delta f$, should also be zero and that the frequency of vibration of the sphere is given by $f=\frac{n}{\pi R} \sqrt{\frac{G^{\prime}}{\rho}}$. This gives a corresponding speed of sound in the material, $c=f \lambda=2 \sqrt{\frac{G^{\prime}}{\rho}}$, which is consistent with the form of the group velocity of acoustic phonons in elastic solids (to within a factor of 2$)^{28}$.

Figure 4 shows plots of $G^{\prime}$ and $G^{\prime \prime}$ values that were calculated using values of $f$ and $\Delta f$ obtained from the droplets. In addition this plot shows measurements of the surface tension $\gamma$ for each solution (see inset) which are used in equations 3 and 4. In each case, the calculated values of $G^{\prime}$ and $G^{\prime \prime}$ obtained from $f$ and $\Delta f$ are plotted against the vibrational frequency of the droplets $f$. These data are compared to the results of shear rheology experiments that were performed on the same solutions using a Kinexus rheometer (Malvern) operating in strain control mode (lines in figure 4). The values of $G^{\prime}$ and $G^{\prime \prime}$ measured using the rheometer were collected at fixed strain amplitudes in the range 1-10\% and no amplitude dependence was observed. Values of $G^{\prime}$ are not given for water in figure 4 because these are extremely small $\left(\sim 10^{-5}\right.$ Pa over the range of frequencies studied) and were difficult to extract in both the shear rheology and droplet vibration studies.

The quantitative agreement between rheology and drop vibration data demonstrates that our model captures the essential physics of vibration of isolated viscoelastic droplets. The scatter in the values obtained for $G^{\prime}$ and $G^{\prime}$ reflects the resolution of the Fourier transform data used to extract $f$ and $\Delta f$, which is fixed by the duration, $T$, of the temporal signals $(T \sim 5 s)$. Obtaining the optimum frequency resolution $(1 / T)$ is a trade off between acquiring data over a long enough period and reducing the contributions of noise from signals that are obtained from heavily damped droplets. Additional sources of error relate to the approximations used for the dispersion relation of capillary waves on a flat, semi-infinite medium and the condition imposed at resonance (see the discussion above). Curvature of the drop is expected to modify the dispersion relation and to introduce an additional source of uncertainty into the values obtained for $G^{\prime}$ and $G$ '. In addition, the finite size of the drops will add a correction to this relation which is likely to be drop size dependent. Moreover, we note that the correct solution to this problem would involve expansion of the 


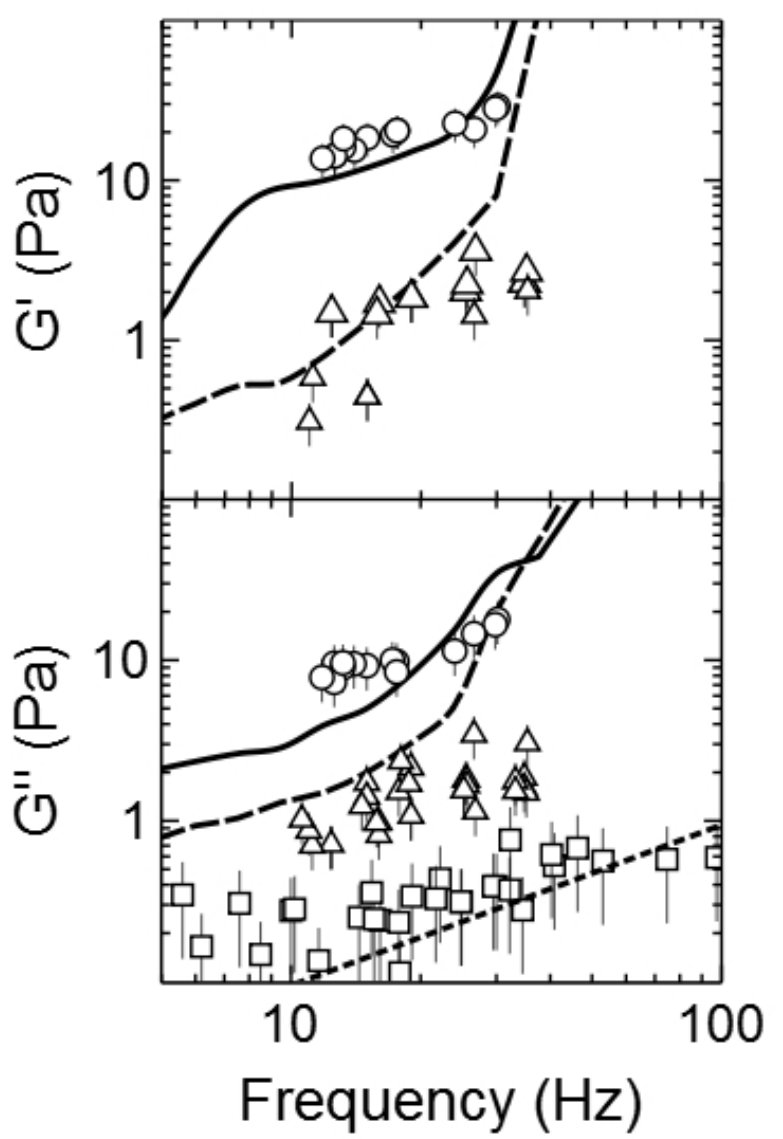

Fig. 4 Frequency dependence of the rheological properties of water and PAA solutions. Data are shown for values of $G^{\prime}$ (top panel) and $G^{\prime \prime}$ (botom panel). The symbols correspond to values obtained from measurements of vibrational frequencies and widths (see text) of droplets of water $(\square), 0.5 \mathrm{wt} \%$ PAA solutions $(\triangle)$ and $1.1 \mathrm{wt} \%$ PAA solutions $(\bigcirc)$. The lines show the results of rheology measurements obtained for the same solutions using a Malvern Kinexus rheometer. spherical harmonics associated with vibrations of an isolated viscoelastic sphere. This would correct the small errors associated with the incorrect mode number dependence discussed above, but has proved to be a challenging task and has not yet been realised. Differences in the near surface rheological properties of the droplets relative to the bulk could also affect the values of $G^{\prime}$ and $G$ ' obtained from the vibration method used here. Such deviations would be expected to be larger in drops with higher surface-to-volume ratios i.e. for smaller drops/higher frequencies.

\section{Conclusions}

A facile optical detection technique was used to measure the vibrational frequencies and spectral widths of diamagnetically levitated water droplets and droplets of water based poly(acrylamide-co-acrylic acid) solutions. Measurements of the size dependence of the frequency and width were used in combination with a heuristic model of viscoelastic drop vibration to extract the frequency dependent rheological properties of the droplets. In the absence of a complete theory of viscoelastic droplet vibration, the heuristic approach adopted here provides a practical alternative for extracting frequency dependent values of $G^{\prime}$ and $G^{\prime}$. The simple measurement technique described above also lends itself to the measurement of liquid properties (e.g. surface tension, viscosity and viscoelastic properties) in a range of different environments (ambient, high pressure, ultrahigh vacuum e.g. ionic liquids) and geometries (e.g. sessile/pendant drops, bubbles, emulsions).

JSS acknowledges support from the LeverHulme Trust (RPG-2012-702). RJAH acknowledges support from an EPSRC fellowship, EP/I004599/1.

\section{References}

1 R. W. S. Rayleigh, Proc. R. Soc. London, 1879, 29, 71-97.

2 S. Chandrasekhar, Proc. London Math. Soc., 1959, 3(9), 141-149.

3 V. Bergeron, D. Bonn, J.Y. Martin and L. Vovelle, Nature, 2000, 405, 772775.

4 D. Bartolo, A. Boudaoud, G. Narcy and D. Bonn,Phys. Rev. Lett., 2007, 99, 174502.

5 B. Qian, M. Loureiro, D.A. Gagnon, A. Tripathi and K.S. Breuer, Phys. Rev. Lett., 2009, 102, 164502.

6 M.I. Smith and V. Bertola, Phys. Rev. Lett., 2010, 104, 154502.

7 B. Vukasinovic, M.K. Smith and A. Glezer, J. Fluid Mech., 2007, 587, 395-423.

8 E.D. Wilkes and O.A. Basaran, Phys. Fluids, 1997, 9(6), 1512-1528.

9 F. Mugele, J.C. Baret,and D. Steinhauser, Appl. Phys. Lett., 2006, 88, 204106.

10 C-Y. Wong and N. Azziz, Phys. Rev. C, 1981, 24(5), 2290-2310.

11 F.F. Pollitz, J. Geophys. Res., 1997, 102(B8), 17921-17941.

12 J. Tromp and J.X. Mitrovica, Geophys. J. Int., 1999, 137, 847-855.

13 S.I. Bastrukov, F. Weber and D.V. Podgainy, J. Phys. G.:Nucl.Part. Phys., 1999, 25, 107-127.

14 T.M. Lee, J. Appl. Phys., 1963, 34(8), 2150. 
15 G.A. Coquin, J. Acoust. Soc. Am., 1964, 36(5), 1052.

16 K. Dobroka, Acta Physica Hungarica, 1983, 54(3-4), 287-295.

17 D.B. Khismatullin, Phys. Rev. E, 2001, 63, 061508.

18 J.C. Chrispell, R. Cortez, D.B. Khismatullin, and L.J. Fauci, Physica D, 2011, 240(20), 1593-1601.

19 H. Akimoto, K. Nagai and N. Sakurai, J. Appl. Mech., 2012, 79(4), 041002.

20 P.G. De Gennes, Scaling Concepts in Polymer Physics, 1979, Cornell University.

21 E. Beaugnon and R. Tournier, J. Phys. III France, 1991, 1, 1423.

22 M.V. Berry and A.K. Geim, Eur. J. Phys., 1997, 18, 307.

23 M.D. Simon and A.K. Geim, J. Appl. Phys., 2000, 87, 6200.

24 R.J.A. Hill, and L. Eaves, Phys. Rev. Lett., 2008, 101, 234501.

25 R.J.A. Hill and L. Eaves, Phys. Rev. E, 2010, 81, 056312.

26 O.I. del Rio and A.W. Neumann, J. Colloid Int. Sci., 1997, 196, 136.

27 H. Pleiner, J.L. Harden and P. Pincus, Europhys. Lett., 1988, 7(5), 383387.

28 C. Kittel, Introduction to Solid State Physics:8th Edition. 2005, Wiley, p94. 